\title{
Ein mythologisches Mosaik aus Syedra/Nauloi
}

Südöstlich des Stadtberges von Syedra, auf dem Gebiet der Unter- bzw. Hafenstadt Nauloi, wurde 1976 bei Bauarbeiten zwischen den Fluren Seki und İshaklı ein Mosaikboden freigelegt ${ }^{1}$. Der ca. 4,95 ×4,54 m große Teil des figürlich, geometrisch und pflanzlich dekorierten Paviments wurde gehoben und ins Archäologische Museum von Alanya gebracht, wo er heute, in Beton vergossen, besichtigt werden kann ${ }^{2}$.

Die schwarzen und weißen Kalksteintesserae der Trennleisten des Mosaiks messen entlang ihrer Oberflächenkanten 1-1,5 cm, während die farbigen Würfel, die in den vegetabilen und figürlichen Darstellungen verwendet wurden, durchwegs kleinere Dimensionen aufweisen. Neben Schwarz und Weiß begegnen Rosa, Hell- und Dunkelrot, mehrere Brauntöne, Grau und Blau sowie vereinzelt Grün. Die Mosaikoberfläche besticht durch eine sehr sorgfältige, enge Setzung der Steinchen, die (vor allem in den Schmuckfeldern) kaum Mörtelfugen erkennen lassen. Über die Bettung und Unterkonstruktion des Tessellats liegen keine Angaben vor.

Das Dekor des Mosaiks verteilt sich über mehrere Zonen. Um ein szenisch geschmücktes Mittelbild fast quadratischen Zuschnitts läuft ein Rahmen aus zwei weißen und einer dazwischenliegenden schwarzen glatten Leiste. Nach außen schließt daran ein schwarzgrundiger Streifen mit einer farbigen, sehr plastischen Akanthusranke an, deren Einrollungen mit Blüten und Früchten verschiedener Formen, aber auch geometrischen Ornamenten (Quadrat, Pelte) gefüllt sind (Abb. 1. 2). Mehrere weiße und schwarze Leisten sowie eine schwarzweiße Sägezahnbordüre (genaue Abfolge der Leisten: weißschwarz-Sägezahn-weiß-schwarz-weiß) trennen den Rankenstreifen von einem figürlich dekorierten Fries, der ursprünglich an allen vier Seiten des Mosaiks vorhanden war. Darauf sind jagende Eroten dargestellt (Abb. 3). An der Außenseite des Frieses verlaufen wieder weiße, schwarze und Sägezahnleisten (weiß-schwarz-Sägezahn-weiß) und eine Bordüre mit einem zweisträhnigen Flechtband auf schwarzem Grund. Die Schlaufen des Flechtbandes sind polychrom und wechseln in regelmäßigen Abständen zwischen Rosatönen und Weiß, Brauntönen und Weiß sowie Grautönen und Weiß. Eine schmale weiße Leiste begrenzte das Mosaik zur ehemaligen Raumwand hin.

Dieser summarischen Schilderung des Aufbaus soll eine detaillierte Beschreibung und Interpretation der einzelnen Bildfelder folgen.

1 Mein Dank gebührt Herrn DI Dr. Gerhard Huber, der mich freundlicherweise zur folgenden Veröffentlichung über das Mosaik eingeladen hat. - Vgl. schon G. HubER, Syedra, Veröffentlichungen der Kleinasiatischen Kommission 6, AnzWien 129, 1992, 27 78 bes. 33. 78. Zum Fundort vgl. ebenda Plan I Nr. VIII. 1. - Zu Nauloi demnächst H. Hellenkemper - F. Hild, Lykien und Pamphylien, TIB 8, s. v.

${ }^{2}$ Vgl. Beitrag G. Huber in diesem Band Abb. 11. - Für eine kurze Vorstellung mit Abbildung, allerdings nicht zutreffende ikonographischer Interpretation des Tessellats vgl. I. Karamut u. a., Alanya Museum, Alanya Turizmini Eski Eserleri ve Müzeleri Sevenler Derneği 1995, 41-43. 
Im Innenbild des Mosaiks sieht man drei weibliche Gestalten, die einen jungen Mann umringen (Abb. 4). Dieser trägt bis unter das Kinn reichende hellbraune Locken, die über der Stirnmitte gescheitelt sind, und ist mit einer braunroten Chlamys bekleidet. Sein rechter Arm ist abgewinkelt und offensichtlich in einem Gestus der Abwehr (mit nach außen gekehrter Handfläche) erhoben, der Mund geöffnet und die Pupillen geweitet. Unmittelbar unter dem Brustansatz bzw. der Achsel ist das Mosaik zerstört. Die drei Frauen - je eine an seinen Seiten, eine weitere hinter ihm -, von denen nur die Oberkörper sichtbar sind, sind nackt bis auf goldene Oberarmreifen, in deren Mitte je ein grüner Edelstein sitzt. Während die mittlere Frau an jedem Arm ein Schmuckstück trägt, besitzen die beiden anderen jeweils nur einen Reifen. Alle drei haben offene, lockige Haare, die am Rücken über die Schultern hinabreichen, bei den beiden seitlichen sind sie hellbraun bzw. dunkelblond, bei der mittleren dunkelbraun. Alle drei tragen darin grüne Schilfkronen. Die mittlere Frauengestalt umfaßt den Mann am Gelenk seiner erhobenen Hand, während die zu seiner Rechten mit ausgestrecktem Arm sein Kinn zu berühren sucht. Aufgrund der schrägen Stellung der Oberkörper scheinen die beiden Frauen zu Seiten des Mannes eine lagernde bzw. liegende Position eingenommen zu haben; dabei stützten sie sich offensichtlich jeweils auf den abgewinkelten Ellenbogen. Zwischen dem Mann und der hinter ihm Stehenden befindet sich ein Gebilde aus hell- und dunkelgrauen Tesserae, bei dem es sich um ein drapiertes Gewand oder einen Felsen handeln könnte. An den Seiten des ansonsten weiß belassenen Hintergrundes existieren Landschaftsangaben: Entlang des rechten Bildrandes sieht man eine dunkelbraune Zone mit unregelmäßigen Konturen, die wohl eine Gesteinsformation darstellt. Über dem Kopf der rechten weiblichen Gestalt ragt ein grüner Zweig schräg ins Bildfeld, auf dem ein weiß-grauer Vogel mit rot umrandetem Köpfchen und gelbem Schnabel sitzt. Am linken Bildrand befindet sich ein weiteres braunes Gebilde, das man im ersten Moment für einen Flügel der Frau oder einen Köcher für Pfeile halten könnte. Allerdings hat man es hier wohl wieder mit dem Teil einer Naturkulisse, in der sich das Geschehen abspielt, zu tun. Fast die Hälfte des unteren Bildteiles ist verloren.

Trotz des fragmentarischen Erhaltungszustandes ist genug vorhanden, um zu erkennen, daß es sich um die Darstellung des Raubes des Hylas durch die Nymphen handelt.

Diese mysische Lokalsage fand Eingang in die Argonautensage: Nachdem die Argo an der mysischen Küste der Propontis gelandet war, wurde der Jüngling Hylas, der schöne Geliebte des Herakles, zum Wasserholen an eine Quelle geschickt. Als er zum Wasser ging, wurden die dort wohnenden Nymphen von seinem Liebreiz so überwältigt, daß sie sich augenblicklich in ihn verliebten. Im Moment, in dem er sich zur Quelle hinabbeugte, um mit dem Krug Wasser zu schöpfen, ergriffen sie ihn und zogen ihn zu sich in die Fluten hinab. Nach einer Version der Erzählung fand Hylas dabei den Tod, nach einer anderen Fassung erlangte er durch das Übergehen in das andere Element die Unsterblichkeit. Jedenfalls konnte er von den Gefährten nicht mehr gefunden werden, sosehr sie ihn auch suchten und nach ihm riefen. Herakles, in maßloser Verzweiflung und Trauer, brach daraufhin seine Teilnahme an der Jagd nach dem Goldenen Vlies ab.

Die Mythenerzählung vom Verschwinden des Hylas wurde in der Antike sowohl in der Literatur als auch in der bildenden Kunst immer wieder rezipiert.

Literarisch belegen läßt sich die Sage bereits im 5. vorchristlichen Jahrhundert durch zwei Fragmente des Logographen Hellanikos von Les- 
bos $^{3}$ : Zum Kult eines verschwundenen Vegetationsgottes oder Jahresköniges im mysischen Kios gehörte das Rufen und Suchen nach ihm ${ }^{4}$. In Vermischung mit der Argonautensage begegnet die Geschichte vom Raub des Hylas als ausgebaute Mythenerzählung erstmals im 3. Jahrhundert v. Chr. bei den Alexandrinern, einerseits bei Apollonios Rhodios im ersten Buch seiner Argonautika $^{5}$, andererseits bei Theokrit, der den Stoff zum ersten Mal für ein eigenes, in sich geschlossenes Gedicht verwendete ${ }^{6}$. Er brachte außerdem als erster Gefühle ins Spiel, nämlich die Liebe des Herakles, das Verliebtsein der Nymphen, schließlich den Schmerz und die Trauer um den Verlust des Knaben.

Damit etablierte sich die Hylasgeschichte als literarischer Stoff und wurde in der Folge noch oft aufgegriffen. Art und Weise sowie Umfang der Rezeption hingen dabei stets von den Absichten des Autors ab: Einerseits wurde die Geschichte in Form einer umfangreicheren Erzählung als Einlage im Argonautenepos (außer bei Apollonios noch bei Valerius Flaccus [wahrscheinlich in den Siebzigerjahren des 1. Jahrhunderts n. Chr. ${ }^{7}$ ] sowie im fiktiven Werk des Orpheus, den in der Spätantike [spätes 4./Anfang 5. Jahrhundert] entstandenen Orphischen Argonautika ${ }^{8}$ ) verwendet, andererseits fand sie ihren Niederschlag in kurzen Anspielungen und Erwähnungen.

Im 13. Idyll des Theokrit steht Herakles im Mittelpunkt der Schilderungen, sein Liebesglück und -leid um den verlorenen Jüngling wird zum zentralen Thema: Die Unzertrennlichkeit zwischen den beiden Männern überschattet alles andere und läßt die Verliebtheit der Nymphen vergleichsweise verblassen. Sie rauben Hylas nicht aus Liebe, werden also nicht als wirkliche Rivalinnen des Herakles, sondern als den Menschen gefährliche Wesen gezeigt.

In ausführlicher Form beschäftigten sich auch Kallimachos (3. Jahrhundert v. Chr.) $)^{9}$ und in seiner Nachfolge Euphorion von Chalkis (wohl zweite Hälfte des 3. Jahrhunderts v. Chr. $)^{10}$ mit dem Hylasstoff; erst Nikander von Kolophon (3. oder 2. Jahrhundert v. Chr.), der eine Sammlung aitiologischer Verwandlungssagen (Heteroioumena) anfertigte, erweiterte die Erzählung um den wesentlichen Punkt, daß Hylas von den Nymphen in ein Echo verwandelt wurde ${ }^{11}$. Properz benutzte den Hylasmythos, um seinen Freund Gallus vor den Gefahren der Rivalität um die Liebe eines Jünglings zu warnen, der dem Hylas an Attraktivität glich: Der Dichter versinnbildlichte die Trauer des verlassenen Liebenden am Beispiel des Herakles. Um die Verfolgung des Geliebten durch die Männerwelt wirksam zu verdeutlichen, erfand Properz zusätzlich die Verfolgung des Hylas durch die Boreassöhne. Die Nymphen aus dem

3 FGrH 4 F 131b = Schol. Apoll. Rhod. 1, 1207; FGrH 4 F 131a = Schol. Apoll. Rhod. 1, 131; zu weiteren - zweifelhaften - frühen Zeugnissen vgl. B. WeBER, Der Hylas des Dracontius, Romulea 2, Stuttgart - Leipzig 1995, 52 Anm. 4.

4 WEBER a. O. 52.

5 Vgl. Roscher ML I 2 (1886-1890) 2792 ff. s. v. Hylas [K. Seeliger]; RE IX (1916) 110 115 s. v. Hylas [E. SitTig].

6 Idyll 13.

7 3, 48-4, 89; vgl. zur Entstehungszeit zuletzt W. EhLERs, Neuere Arbeiten zur Datierung und Überlieferung der Argonautica des Valerius Flaccus, in: M. KoRN - H. J. Tschiedel (Hg.), Untersuchungen zu den Argonautica des Valerius Flaccus, Spudasmata 48, Hildesheim 1991, 17-24.

8 Die Hylaserzählung umfaßt darin 29 Verse (629-657); vgl. WeBer a. O. 59.

9 Kall. frg. $596=$ Schol. Apoll. Rhod. 1, 1207.

10 WeBer a. O. $64 \mathrm{f}$.

11 Die Sammlung ist in Auszügen in Prosa beim Mythographen Antoninus Liberalis (2. Jahrhundert n. Chr.) überliefert: Hylas (met. 26); vgl. WEBER a. O. 65. 
Mythos stehen hingegen für die Frauen, die Gallus' Jüngling in den Bädern verführen wollten ${ }^{12}$.

Ausführliche Behandlung erfuhr der Stoff danach erst wieder im 4. Jahrhundert durch Ausonius, der zwei Epigramme über Hylas verfaßte ${ }^{13}$. Interessanterweise spielte er bei seinen Betrachtungen über vergebliche oder nicht erwiderte Liebe die Beziehung zwischen Hylas und den Nymphen in den Vordergrund, während Herakles als Liebhaber nicht vorkommt.

Im sogenannten Codex Salmasianus der Anthologia Latina, befindet sich ein Epigramm über Hylas, das gegen Ende der Vandalenherrschaft in Nordafrika geschrieben worden ist ${ }^{14}$ : Im ersten Gedicht wird das übergeordnete Thema der Epigrammreihe deutlich, das man als „Macht des Zufalls über den Menschen" umschreiben könnte ${ }^{15}$. Als Beispiele aus dem Bereich der Sage werden Hylas, Herakles und die Nymphen als Opfer des fatum gezeigt, während konkrete Motive - wie der Raub und die Suche nach Hylas - ausgeblen$\operatorname{det} \operatorname{sind}^{16}$.

Ende des 5. bzw. Anfang des 6. Jahrhunderts hat der in Karthago lebende Dichter Dracontius in seiner Gedichtsammlung der Romulea (2) der Hylasgeschichte breiten Platz eingeräumt. Dabei behielt er zwar die Hauptmotive der seit dem Hellenismus populären Mythenerzählung bei, allerdings geriet die Liebe des Herakles in den Hintergrund, während die plötzlich entbrannte Liebe der Nymphen herausgestrichen wurde. Obwohl der Knabe Hylas das Opfer einer Gottheit wird, geht es für ihn insofern glücklich aus, als er die Unsterblichkeit erlangt ${ }^{17}$.

Neben den angeführten literarischen Langversionen der Hylasgeschichte lebte diese seit dem Hellenismus auch in Anspielungen und kurzen Erwähnungen bis in die Spätantike fort. In den meisten Fällen wurden Elemente der Erzählung herausgegriffen, um anhand davon mythologische Vergleiche anzustellen. Andererseits wurde die Geschichte auch weiterhin ausführlicher dargestellt, allerdings stets unter einem bestimmten Blickwinkel, der der Absicht des Autors entsprach. Eines der wichtigsten Motive war dabei die Schönheit und Anziehungskraft des Hylas ${ }^{18}$, die als Vergleich für zeitgenössische Personen, meist Lieblingsknaben sozial hochgestellter Persönlichkeiten oder sogar des Kaisers diente ${ }^{19}$. Exemplarisch verwendet wurden auch die Liebesbeziehung zwischen Herakles und Hylas ${ }^{20}$, Hylas' Tod bzw. seine Opferrolle $^{21}$. Die verzweifelte Suche und das vergebliche Rufen nach Hylas, das viele

12 Prop. 1, 20; Weber a. O. 66-68; H. Diller, Herakles und Hylas - Theokrit und Properz, in: E. LeFÈVRE (Hg.), Monumentum Chiloniense. Studien zur augusteischen Zeit. Festschrift für E. BRUCK, Amsterdam 1975, 419-431 bes. 427.

13 Epigrammata de diversis rebus 97-105.

14 Vgl. W. Schetter, Zum anonymen Libellus epanaleptischer Monodisticha des Salmasianischen Corpus, Hermes 114, 1986, 231-239 bes. 238f.

15 vgl. Anth. Lat. 38R = 255B: De fortuitis casibus: Omnia casus agit: fatum consulta sequuntur./ cedamus fatis: omnia casus agit.

16 WEBER a. O. 70

17 Für die Funktion des öffentlich verlesenen Gedichts zur Bewahrung und Vermittlung traditionellen Bildungsgutes vgl. WEBER a. O. 254f. mit Diskussion abweichender Deutungen.

18 z. B. bei Apoll. Rhod.; Theokr., Prop., Ov., Val. Fl.; vgl. Weber a. O. 72-82.

19 Vgl. Stat. silv. 2, 1; 3, 4; Mart. 5, 48; 9, 65; Hyg. fab. 271; Lukian. 2, 4-29; Philostr. II. Her. 26, 2-4.

20 Apoll. Rhod., Theokr., Prop., Val. Fl., Stat., Mart., Plut., Lukian., Pap. Oxyr. 3723 (2. Jahrhundert), Corpus Philostrateum; vgl. WeBER a. O. 82-93.

${ }^{21}$ Die Gleichsetzung von Raub und Tod begegnet erst in der kaiserzeitlichen Literatur: Sen. Med. 646-651. Andere Autoren streichen die Opferrolle, den unverdienten frü- 
Autoren besonders betonten, wurde geradezu zum Synonym für vergebliche Suche $^{22}$.

Der Hylasstoff eignete sich als gängiges, vielleicht sogar „triviales“ Thema auch zur Abgrenzung anderer literarischer Inhalte und Gattungen ${ }^{23}$, in historiographischen Texten, Reisebeschreibungen und enzyklopädischen Werken floß er in seiner aitiologischen Funktion für den Kult aber auch für die Benennung von Orten in Mysien in die Fachliteratur ein ${ }^{24}$. Christlichen Autoren diente Hylas als Beispiel für die Verirrung homoerotischer Liebe, auch um die Frevelhaftigkeit heidnischer Götter(geschichten) aufzuzeigen ${ }^{25}$.

Als Abschluß dieses ausführlichen Exkurses zum Hylasmythos und seiner Rezeption in der Literatur sei auf seine Verwendung als Gegenstand der bildenden Kunst in literarischen Texten hingewiesen: In den Satyrica des Petron wird der eben von seinem Geliebten zugunsten eines anderen verlassene Encolpius geschildert, der in einer Bildergalerie Ablenkung von seinem Schmerz sucht ${ }^{26}$. Dort sieht er drei Bilder des Malers Apelles, deren gemeinsames Thema die homoerotische Liebe zwischen Paaren aus der Mythologie ist. Zwischen der Entführung des Ganymed und der Verwandlung des Hyakinthos sieht er ein Gemälde mit Hylas und einer Nymphe. Es ist nicht klar, ob es sich bei Petron um eine fiktive oder eine reale Bildbeschreibung handelt: Die „Anordnung“ der Bilder (links Ganymed, Mitte Hylas, rechts Hyakinthos) könnte ein literarischer Kunstgriff sein, der mit dem Inhalt der einzelnen Mythenerzählungen korrespondiert. Nur die Hylasgeschichte vereint - gleichsam als Mittelstück - die Aspekte der beiden anderen Geschichten in sich: Ein Dritter hat von der geliebten Person Besitz ergriffen und der Moment des Verlustes wird gezeigt ${ }^{27}$.

Es ist aber auch denkbar, daß das in den Satyrica beschriebene Gemälde des Hylasraubes auf real existierende Kunstwerke Bezug nimmt. Analog zur Literatur erfreute sich der Mythos nämlich in der dekorativen Kunst, besonders in der häuslichen Bilderwelt, wahrscheinlich ab dem Hellenismus einer gewissen Beliebtheit. Das älteste bekannte Bildwerk stammt zwar erst aus dem 1. Jahrhundert v. Chr., allerdings sind ältere Vorlagen dafür zu vermuten:

Dieses Hylasbild ist ein Mosaikemblem und stammt aus einer Villa in Tor Bella Monaca bei Rom ${ }^{28}$. Darauf sieht man den jungen Helden mit Speer, Schwert und Krug gerüstet zur Quelle gehen, als eine aus dem Wasser auftauchende Nymphe versucht, ihn am Arm zu sich hinabzuziehen. Drei ihrer Schwestern lagern um die Quelle, beteiligen sich allerdings nicht am Geschehen.

hen Tod, aber auch die Erlangung der Unsterblichkeit heraus: vgl. Ov. ars 2, 110; Val. Fl. 4, 23. 26-27; Stat. silv. 1, 5; Mart. 6, 68; Philostr. II Her. 45, 6; Auson. epigr. 97.98 und WeBER a. O. 102-108.

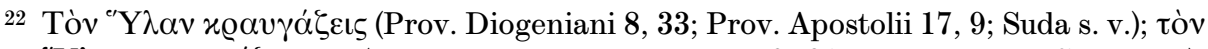

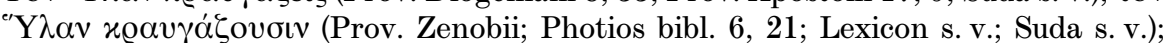

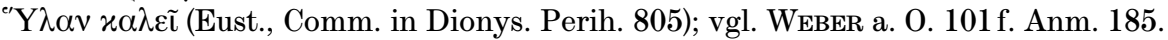

${ }^{23}$ Verg. georg. 3, 1-13; Ov. trist. 2, 207; Mart. 10, 4; Iuv. 1, 1-18. 52-55; Philostr. II Her. 45,6 .

24 WEBER a. O. 117-122.

25 Clem. Alex. protrept. 2, 33, 5 ; Arnob. 4, 19-31; Firm.; Pseudoklementinen, Homilie 5, $15,1-3$; Tert. nat. $2,14,7$; Prud. $1,116-121$.

$2683,1-2 ; 3-5$.

27 WeBer a. O. 114f. Anm. 225.

28 W. Widrig, AJA 91, 1987, 314f.; A. LA Regina (Hg.), Museo Nazionale Romano. Palazzo Massimo alle Terme, Rom 1998; S. MUTH, Erleben von Raum - Leben im Raum. Zur Funktion mythologischer Mosaikbilder in der römisch-kaiserzeitlichen Wohnarchitektur, Archäologie und Geschichte 10, Heidelberg 1998, Taf. 42, 1. 
Diese bisher singuläre Version enthält ein Element, durch das sich in der Folge alle Hylasdarstellungen der bildenden Kunst von denen der Literatur wesentlich unterscheiden: Hylas wird als erwachsener Mann gezeigt, nicht als Jüngling.

Bei einem anderen Darstellungstypus dringen die Nymphen von einer Seite auf Hylas ein, während dieser erschrocken vor ihnen zurückweicht: Vertreter dieser Version sind pompejanische Wandgemälde aus der Domus Sexti Pompei Axiochi (VI 13, 19) aus claudischer ${ }^{29}$ sowie aus der Casa delle Forme di Crete (VII 4, 62) aus flavischer Zeit ${ }^{30}$.

Ein flavisches Stuckrelief aus der Casa di Meleagro in Pompeji (VI 9, 2) zeigt wiederum eine einzigartige bildliche Umsetzung der Geschichte: Hylas läuft mit Wasserkrug und Speer auf die Quelle zu; eine Nymphe sitzt auf einem Felsen und wendet sich ihm zu, ohne ihn jedoch zu berühren ${ }^{31}$.

Der gängigste Darstellungstyp, der auch nach der frühen Kaiserzeit in der Wohnhausdekoration verbindlich bleibt, ist derjenige, bei dem Hylas von allen Seiten von den Nymphen bedrängt wird. Er erscheint zum ersten Mal in Pompeji auf einem augusteischen Wandgemälde (IX 7, 16) ${ }^{32}$, danach auf einem pompejanischen Wandbild claudischer Zeitstellung (I 7, 19) ${ }^{33}$, schließlich auf einem flavischen Wandmosaik aus einem Wohnhaus unter der Caserma dei Corazzieri in Rom ${ }^{34}$

Die Hylassage, vor allem die Szene seines Raubes durch die Nymphen, wurde in der Folge während der römischen Kaiserzeit noch oft bildlich umgesetzt. Bei den meisten Exemplaren handelt es sich um Mosaikfußböden aus Wohnhäusern, besonders im Westen des Reiches. Außer einem Beispiel aus Vienne, welches dem ausgehenden 2 . oder dem frühen 3. Jahrhundert zugeschrieben wird ${ }^{35}$, stammen die bekannten Tessellate aus Nordafrika und Spanien: Drei Böden werden ins 2. oder 3. Jahrhundert datiert ${ }^{36}$, die anderen

${ }^{29}$ LIMC V 1 (1990) 574 ff. s. v. Hylas Nr. 9 [J. OAKLEY]; R. Ling, MEFRA 91, 1979, 773 ff. bes. 777 Nr. 3; 778 Abb. 1; I. Baldassare - T. Lanzilotta - S. Salomi (Hg.), Pompei. Pitture e mosaici V, Regio VI, parte 2, Rom 1994, 223 Abb. 38.

${ }^{30}$ LIMC a. O. Nr. 10*; Ling a. O. 778 f. Nr. 4 Taf. 3; I. Baldassare - T. LanzilotTa - S. SALOMI (Hg.), Pompei. Pitture e mosaici VII, Regio VII, parte 2, Rom 1997, $151 \mathrm{f}$. Abb. 18f.; Muth a. O. Taf. 42, 4.

31 LIMC a. O. Nr. 27; Ling a. O. 781 Abb. 2, 9; 782 Nr. 9; I. Baldassare - T. LanzilotTa S. Salomi (Hg.), Pompei. Pitture e mosaici IV, Regio VI, parte 1, Rom 1993, $684 \mathrm{f}$. Abb. 53.

${ }^{32}$ LIMC a. O. Nr. 7*; Ling a. O. 776 Nr. 1 Taf. 1; K. Schefold, Die Wände Pompejis. Topographisches Verzeichnis der Bildmotive, Berlin 1957, 268.

33 LIMC a. O. Nr. 8; Ling a. O. 776 f. Nr. 2 Taf. 2; I. Baldassare - T. LanzilotTa - S. SALOMI (Hg.), Pompei. Pitture e mosaici I, Regio I, parte 1, Rom 1990, 763ff. Abb. 24. 27. 29; Muth a. O. Taf. 42, 3.

34 vgl. E. M. Steinby (Hg.), Lexicon Topographicum Urbis Romae III, Rom 1996, 82ff. Abb. 53; F. CoARELLI, Roma sepolta, Rom 1984, 151 ff. bes. 152f. Abb.; M. DE Vos, Late Republican and Flavian wall mosaics in the Horti Sallustiani (Rome), in: VI Coloquio Internacional sobre Mosaico Antiguo, Palencia - Mérida 1990 (1994) 91-96 bes. 93 Abb. 1; Muth a. O. Taf. 42, 2.

35 LIMC a. O. Nr. 13; LING a. O. 803 Nr. 13; J. LANCHA, L'iconographie d'Hylas dans les mosaiques romaines, in: III Coloquio Internazionale sul Mosaico Antico, Ravenna 1980 (1983) 381-392 bes. 383 Abb. 2.

36 Cirta (Constantine), Algerien: zweite Hälfte 2. bis 3. Jahrhundert; vgl. Muth a. O. 101. 361 f. A 9 Taf. 8, 3; K. M. D. Dunbabin, The Mosaics of Roman North Africa. Studies in Iconography and Patronage, Oxford 1978, 178. 255 Constantine Nr. 5 Taf. 48 Abb. 174; Ling a. O. 804 Nr. 18; 814 Taf. 9; LIMC a. O. Nr. 16*. - Italica, Casa de Hylas: zweite Hälfte 2. bis frühes 3. Jahrhundert; vgl. MutH a. O. 101. 432 f. H 21 Taf. 7 , 2-3; LING a. O. 803 Nr. 1; LIMC a. O. Nr. 12* - Volubilis, Maison à la mosaique de Ve- 
drei in die Spätantike ${ }^{37}$. Eine opus-sectile-Wandverkleidung aus der Basilika des Iunius Bassus in Rom von $331 \mathrm{n}$. Chr. zeigt ebenfalls den Raub des Hylas durch die Nymphen ${ }^{38}$. Diesen westlichen Beispielen, konnte bisher nur ein Mosaik aus dem Osten des Imperium Romanum gegenübergestellt werden. Es handelt sich um ein Paviment aus einer römischen Villa von Amphipolis, das in die zweite Hälfte des 2. oder an den Beginn des 3. Jahrhunderts datiert wurde $^{39}$ : Hylas, dessen Name über seinen Kopf geschrieben ist, wird fast frontal gezeigt, das linke Knie ist abgewinkelt und auf einen Felsblock gestützt, während das rechte Bein gerade zu Boden gestreckt ist. Den rechten Arm in abwehrendem Gestus erhoben, wendet er den Blick von der Nymphe zu seiner Linken ab, die gierig nach seinem Hals greift. Hylas trägt nur eine Chlamys, der Wasserkrug ist ihm entglitten. Hinter ihm links und zu seiner Rechten befindet sich je eine weitere, ihn bedrängende Nymphe.

Ein Wandgemälde aus den Thermen von Salamis auf Zypern hingegen, das von seinem Entdecker V. KARAGEORGHIs als Hylasraub gedeutet wurde, konnte zuletzt von J. BALTY überzeugender als Darstellung der badenden Artemis, die von Aktaion beobachtet wird, identifiziert werden ${ }^{40}$.

Auch nur vermeintlich handelt es sich bei dem in J. Lanchas Liste angeführten Beispiel um ein Hylasmosaik aus Seleukeia aus Pierien ${ }^{41}$ : Das Paviment, das sich in Wirklichkeit in Seleukeia in Pamphylien befindet, zeigt vielmehr Orpheus und die Tiere und im Rahmen dessen eine Darstellung der Hyle, die Personifikation des Waldes (mit Namensbeischrift).

Man wird also vorläufig festhalten müssen, daß der Hylasmythos im Westen wahrscheinlich häufiger bildlich umgesetzt worden ist als im Osten. AuBerdem läßt sich ein starker Überhang der Darstellungen im Kontext von

nus, Marokko: Ende 2. bis erste Hälfte 3. Jahrhundert; vgl. Muth a. O. 101. 402-405 A 45-1 Taf. 6, 4; Ling a. O. 804 Nr. 16; DunBabin a. O. 86 f. 277 Volubilis Nr. 2 c; LIMC a. O. Nr. $17^{*}$.

37 Quintana del Marco, Villa de Los Villares, Spanien: spätes 3. bis 4. Jahrhundert; vgl. Muth a. O. 101. 439 f. H 28 Taf. 8, 1; Ling a. O. 804 Nr. 14; LIMC a. O. Nr. 14; Lancha a. O. 382. 384 Abb. 3. - Cuicul (Djemila), Maison d'Hylas, Algerien: zweite Hälfte 4. bis frühes 5. Jahrhundert; vgl. Muth a. O. 101. 363f. A 12 Taf. 8, 2; Dunbabin a. O. 44 mit Anm. 36; 256 Djemila Nr. 5; Ling a. O. 805 Nr. 22; 816 Taf. 13; LIMC a. O. Nr. 19*. Carranque, Villa de Materno, Spanien: zweite Hälfte 4. bis frühes 5. Jahrhundert; vgl. Muth a. O. 101. 415-419 H 7-3 Taf. 5, 1. 3; LIMC a. O. Nr. 20.

38 LIMC a. O. Nr. 18; Ling a. O. 805 Nr. 20 Taf. 11; R. Bianchi Bandineldi, Rom - Das Ende der Antike, München 1971, 293 Abb. 270.

39 Vgl. E. G. Stikas, Prakt 1975, 1, Textplan E Taf. 75b. 76b. 77; BCH 100, 1976, 686 Abb. 233; A. KanKeleit, Kaiserzeitliche Mosaiken in Griechenland II, Bonn 1994, $3 \mathrm{ff}$. Nr. 2.

40 V. Karageorghis, Anaskaphai Salaminos, 1964-1966, RDAC 1966, 13-19 bes. 15 Taf. 7, 2; J. Balty, RDAC 1988, 2, 266; D. Michaelides, Cypriot Mosaics, Nikosia 1987, Taf. 13; DERs., RDAC 1987, Taf. 62, 1: Die Malerei in der Konche über dem südlichen Eingang in das mittlere sudatorium des Bades zeigt einen Jüngling in Chiton und Chlamys, der einen Speer in der Linken hält. Rechts von ihm sieht man Kopf und rechten Arm einer Frau mit Diadem und Armreif, die bis zu den Schultern im Wasser steht. LANCHA a. O. 383 Abb. 1 hat die Hylasdeutung aufgegriffen, allerdings unverständlicherweise Hylas als die Person im Wasser bezeichnet. - Die Malerei besitzt einen terminus ante quem in dem Erdbeben von 342 n. Chr., welches das Bad zerstörte, dürfte aber nicht viel früher (zu Beginn des 4. Jahrhunderts?) entstanden sein; vgl. LIMC a. O. Nr. 43.

41 LANCHA a. O. $381 \mathrm{ff}$. bes. 382 Tabelle A; falsch übernommen in LIMC a. O. Nr. 46. Zum Orpheusmosaik mit der Hyledarstellung aus dem pamphylischen Seleukeia vgl. M. MeldinK, AJA 80, 1976, 273 (J. INAN); F. TüLeK, Efsuncu Orpheus/Orpheus the Magician, Istanbul 1998, 50f. Abb. 25-26. 
Wohnhäusern erkennen: Von den 24 im LIMC angeführten Beispielen stammen allein 13 aus Privathäusern ${ }^{42}$, während sich die übrigen auf Thermen ${ }^{43}$, andere öffentliche Gebäude ${ }^{44}$ sowie auf den Grabbereich ${ }^{45}$ verteilen. Das zahlreiche Vorkommen des Hylasraubes in der häuslichen Bilderwelt ${ }^{46}$ hat zu den unterschiedlichsten Deutungen der Intentionen der Auftraggeber Anlaß gegeben. Bevor wir darauf näher eingehen, sei vorausgeschickt, daß schon die ausschließliche Wahl der Raubszene für das Ausstattungsprogramm des Privathauses zeigt, daß hier mit dem Hylasstoff anderes beabsichtigt war als in der Literatur. Dort erwiesen sich - vom Hellenismus bis in die Spätantike und trotz aller Verschiedenheiten im Einzelnen - bestimmte Erzählmotive als Konstanten: Dazu zählen die Liebe des Herakles (das homoerotische Ideal von Erastes und Eromenos), die Schönheit und Attraktivität des jungen Hylas, das Liebesverlangen der Nymphen, der Raub durch diese, der Verlust des Geliebten sowie die Suche nach ihm. In den meisten schriftlichen Quellen wurde die Konkurrenz zwischen Herakles und den Nymphen um den Geliebten zum zentralen Thema, gleichzeitig der Tod bzw. der Ausweg der Verwandlung oder Apotheose. Bis in die Spätantike nahmen die Nymphen keine wesentliche Stellung in der Erzählung ein, sondern dienten gleichsam als Folie, vor der sich jene entwickelte. Zusammenfassend läßt sich sagen, daß Tragik durch außergewöhnliche Schönheit und homoerotische Liebe die wesentlichen Elemente in der literarischen Rezeption der Hylassage waren, während die Liebe zwischen Mann und Frau außer bei Dracontius nicht betont wurde.

In der bildenden Kunst scheint im Gegensatz dazu die Hervorhebung von anderen Motiven beabsichtigt gewesen zu sein, und das parallel zur (= unbeeinflußt von der) Literatur bereits seit der frühen Kaiserzeit ${ }^{47}$ : Stets ist der Moment des Raubes durch die Nymphen gezeigt, hingegen niemals die Liebe zwischen Herakles und Hylas, auch nicht die verzweifelte Suche des Ersteren nach dessen Verschwinden. Andererseits wurde in den Gemälden und Mosaiken auch nie das Leben des Hylas im Jenseits mit den Nymphen ins Bild gebracht: Aus diesem Grund wird man von einer eschatologischen Deutung der Darstellungen im Wohnbereich Abstand nehmen müssen ${ }^{48}$. Zwei extreme Po-

42 Noch nicht genannt sind hier die Hylasbilder aus den Wohnhäusern von Tor Bella Monaca (s. o.) und unter der Caserma dei Corazzieri (s. o.).

43 LIMC a. O. Nr. 2. 25. 26. 28*.

44 Nr. 18 ?

45 Nr. 23. $24^{*} .30^{*}$.

46 In Spanien und Nordafrika sind nur Europa, Orpheus, Leda und Ganymed häufiger auf Mosaiken im häuslichen Bereich abgebildet. Hingegen kommen Einzeldarstellungen großer Helden wie Achill, Herakles, Bellerophon und Meleager seltener vor; vgl. Muth, Erleben im Raum 100 Anm. 334.

47 Eine ausführliche Diskussion der Interpretationsansätze bietet MUTH a. O. 102-106.

48 Bezeichnenderweise sieht man Hylas unter den Nymphen im Jenseits hingegen in der Sepulkralkunst; vgl. Via Flaminia, Tomba dei Nasoni (Mitte 2. Jahrhundert), Nischenmalerei Ling a. O. Nr. 32 Taf. 14, B. AndREAe, Studien zur römischen Grabkunst, Berlin 1963, 123 Taf. 52. - Zum Inventar eines Grabes aus Concesti (Rumänien) gehört eine silberne Situla mit dieser Darstellung (zweites Viertel 4. Jahrhundert); vgl. Ling a. O. Nr. 33; L. Matzulewitsch, Byzantinische Antike (1929) $134 \mathrm{f}$. Taf. 45; G. TüRK, Zu den Darstellungen des Hylas, JdI 12, 1897, 86-91 Taf. 4. 5. LANCHA a. O. 381-392 interpretierte den Hylasraub vor allem in Verbindung mit dionysischen Szenen bzw. den Mitgliedern seines Thiasos wie Eroten als Warnung vor Fehlverhalten in Liebesdingen. - Tod und Verwandlung als zentrale Themen der mit dem Hylasraub verbundenen Bildensembles deuteten D. Fernández-Galiano - B. PÁTON LoRca - C. M. BAtalla Corchenilla, in: VI Coloquio Internacional sobre Mosaico 
sitionen bei der Interpretation der häuslichen Hylasdarstellungen - entwikkelt anhand der pompejanischen Bilder - vertraten K. SCHEFOLD ${ }^{49}$ einerseits, R. $\mathrm{LING}^{50}$ andererseits, beide jedoch unter Annahme der Existenz eines Gesamtkonzepts der bildlichen Raumausstattung: Während der eine in den Hylasszenen Sinnbilder der Apotheose, zugleich ein warnendes Exemplum für Frevler und Vermessene, aber auch die Illustration tragischer Liebe sehen wollte, sprach LiNG den Bildern jeglichen tieferen Inhalt ab und erklärte sie zu Objekten reinen "picture collectings“.

J. Arce hat mit der Deutung der Hylasbilder als Feier von Liebe und Leidenschaft wahrscheinlich den richtigen Weg der Interpretation eingeschlagen, wenn er auch in diesem Zusammenhang die Funktion des cubiculum als Schlafzimmer zu eng aufgefaßt hat ${ }^{51}$.

S. Muth konnte verdeutlichen, daß die Interpretation eines Mythenbildes niemals ihren Ausgang von der umgebenden Architektur nehmen darf, da das zwangsläufig zu einseitigen Auslegungen führt ${ }^{52}$. Vielmehr muß die Deutung für den Kontext des Raumes erfolgen. Dabei ist davon abzugehen, eine Idee hinter dem gesamten Ausstattungsprogramm zu vermuten, deren Illustration alles untergeordnet ist. Da die Räume des antiken Wohnhauses nicht auf bestimmte Funktionen (Essen, Schlafen, etc.) reduziert werden dürfen, sondern bestenfalls Bereichen mit mehr öffentlich-repräsentativem Charakter (z. B. oecus, triclinium) und Bereichen mit eher zurückgezogen-privatem Charakter (cubiculum) zuzuordnen sind, kann die bildliche Ausstattung auch nur zur Erkennung des Ambientes dienen, in dem sich ein Raum befindet.

Die bekannten Hylasraubmosaiken der römischen Wohnhäuser schmükken durchwegs kleine oder mittelgroße Räume, die, wenn auch nicht in allen Fällen die Gesamtanlage freigelegt worden ist, den „privaten“53 Lebensbereichen angehören ${ }^{54}$. Mancherorts gibt es eine Kombination mit anderen Mythenillustrationen ${ }^{55}$. Wenn man davon ausgeht, daß das Haus unter anderem eine Bühne der Selbstdarstellung seiner Bewohner, vor allem des dominus und der domina war, auf der diese nicht nur agierten, sondern sich auch entsprechend inszenierten, erscheint es plausibel, mit S. MUTH anzunehmen, daß die Mythenbilder für diese Zwecke eingesetzt wurden: Als Gegenpol zu Männeridealen wie virtus, munificentia und paideia, die in den Dekorationen der

Antiguo, Palencia - Mérida 1990 (1994) 322 ff. bes. 324 . 326 ausgehend vom Hylasmosaik in der Villa von Carranque s. o.

49 K. Schefold, Pompejanische Malerei, Basel 1952, 131. 187; DERs., Vergessenes Pompeji, München 1962, 88. 98.

50 LiNG a. O. $800 \mathrm{ff}$.

51 J. Arce, MM 27, 1986, $372 f$.

52 Muth a. O. 109.

53 Zur Problematik der Anwendung moderner Begriffe wie „privat“ und „öffentlich“ auf die Räume des antiken Wohnhauses vgl. ebenda 53 Anm. 154; 130 Anm. 457.

54 Ebenda 130-134. In der Villa de Materno bei Carranque bezeichnet eine Inschrift auf dem Schwellmosaik den Raum als cubiculum. - Eine Ausnahme stellt lediglich das Mosaik aus der Maison d'Hylas in Cuicul dar, das den Boden einer kleinen Portikus schmückt. In unmittelbarer Nähe des Hylasbildes befindet sich ein Wasserbecken. Möglicherweise war der gemeinsame Aspekt des Wassers ausschlaggebend für die Anbringung des Mythenbildes.

55 z. B. Carranque, Villa de Materno: Artemis und Aktaion, Amymone und Poseidon, Pyramus und Thisbe. - In der Maison à la mosaique de Vénus in Volubilis sind in den seitlichen Friesen des Hylasbildes Eroten in Zirkusszenen gezeigt, während das Mosaik im benachbarten Raum die badende Artemis unter den Nymphen zeigt; vgl. MuTH a. O. 133 Taf. $29,1$. 
Räume, in denen man Gäste und Klienten empfing, durch Zirkus-, Jagd- und Szenen aus dem Domänenalltag veranschaulicht wurden, evozierten Bilder mythologischer Liebespaare den Aspekt männlicher Attraktivität. Es geht zu weit, die Bilder unmittelbar mit der sexuellen Aktivität des Hausherrn zu verbinden, doch wurde durch jene eine Atmosphäre geschaffen, die der des offiziell-geschäftigen Alltagslebens entgegenstand: Das Umfeld dieser Darstellungen ist das otium, durchaus mit erotischer Komponente, jedenfalls heitere Gelassenheit und Lebensfreude abseits der Geschäfte. Vielleicht erklärt sich die Präferenz bestimmter mythologischer Darstellungen in der Bilderwelt des Hauses daraus, daß sich der dominus in seiner erotischen Attraktivität - als Liebhaber bzw. Geliebter - feiern ließ und sich dafür besonders das Motiv des „ergriffenen Mannes“ eignete $^{56}$ : Außer Hylas, der von den Nymphen geraubt wird, sind hier auch andere Raub- und Verfolgungsszenen aus der Mythologie, z. B. Satyr und Nymphe, Achill und die Töchter des Lykomedes denkbar $^{57}$. Wichtig ist allerdings die Anwesenheit von Mann und Frau, während homoerotische Liebe anscheinend nicht ins Bild gebracht wurde. Auch die Umformung des Jünglings Hylas aus der Sage und Literatur in einen attraktiven Mann erhält unter diesem Gesichtspunkt Plausibilität. Nicht unerwähnt bleiben soll in diesem Zusammenhang, daß auch mythologische Vorbilder bzw. Symbole für Schönheit und Anziehungskraft der Hausherrin existierten, die oft parallel zu denen des dominus umgesetzt wurden. Das Erscheinungsbild der mythologischen Gestalten wurde demgemäß auch manchmal demjenigen der lebenden Personen angenähert ${ }^{58}$.

Was kann man aus dem Gesagten für das Mosaik aus Nauloi ableiten? Sein genauer Fundort, geschweige denn sein architektonischer Kontext sind nicht bekannt. Allerdings engt sich m. E. aus den oben stehenden Ausführungen der Kreis seiner möglichen Anbringung stark ein. Ich schlage vor, den Boden einem Wohnhaus zuzuordnen, zumal die Hylasmosaiken aus öffentlichen Gebäuden entweder nicht die Raubszene zeigen ${ }^{59}$ bzw. nicht gesichert sind.

Ein wesentliches Element für die Interpretation der architektonischen Umgebung des Mosaiks aus Nauloi ist der ursprünglich vierseitige Rahmenstreifen mit jagenden Eroten: Unterhalb der Hylasszene ist er von außen her zu betrachten, während die Füße der Tiere und Eroten an den anderen Seiten auf die Mosaikmitte ausgerichtet sind. Das Dekor des Schmuckbodens war daher eindeutig für einen Betrachter konzipiert, der von vorne, wohl beim Betreten des Raumes, auf das Bild schaute. Im Erotenfries ändert sich die Bewegungsrichtung an jeder Seite, er war demnach nicht als umlaufende Komposition geplant. Während Tiere und Eroten oberhalb der Hylasszene von

56 S. Muth, Hylas oder „Der ergriffene Mann“: Zur Eigenständigkeit der Mythenrezeption in der Bildkunst, in: F. DE Angelis - S. Muth (Hg.), Im Spiegel des Mythos. Bilderwelt und Lebenswelt/Lo specchio del mito. Immaginario e realtà. Symposium, Rom 19.-20. Februar 1998, Palilia 6, Wiesbaden 1999, 109-129.

57 Muth, Erleben von Raum 123f. 151 f. Anm. 552.

58 Die Nymphen des Hylasmosaiks aus Quintana del Marco tragen z. B. Diademe statt der üblichen Schilfkronen, in Cuicul zeitgenössische Frisuren vornehmer Frauen; vgl. J. Blazquez, ArchEspA 50/51, 1977/78, 282 Abb. 1; Muth a. O. Taf. 8, 1.2.

59 Auf dem Hylasmosaik der Thermen südwestlich des Leuchtturmes in Thaenae (Henchir Thina, Tunesien) sieht man nur den Protagonisten an der Quelle knien; LIMC a. O. Nr. 2 (spätes 3. Jahrhundert). - Allerdings zeigen frühkaiserzeitliche Stuckreliefs aus Thermenanlagen den Moment des Raubes: Baiae, Venusthermen LIMC a. O. Nr. 25; Pompeji, Terme del Sarno LIMC a. O. Nr. 26; Pompeji, Stabianer Thermen LIMC Nr. 28*. 
rechts nach links eilen, bewegen sie sich an der rechten Seite von links nach rechts (= von oben nach unten) und in die Gegenrichtung, während sie an der Unterseite des Mittelbildes von links nach rechts jagen. Es ist zu vermuten, daß die Bewegungsrichtung an der nicht erhaltenen linken Seite des Mosaiks gegengleich zur gegenüberliegenden Seite verlief ${ }^{60}$. Der Bildgrund ist weiß bis auf die grauen Schattenstreifen zu Füßen der Figuren und die wenigen Bäume, die die einzigen Landschaftsangaben darstellen.

Die geflügelten Eroten tragen kurze, verschiedenfarbige Tuniken, enge Hosen und Schuhe. Bei der Beschreibung der Szenen beginnen wir an der rechten Seite oben (Abb. 3. 5): Einer der Eroten in roter Tunika, grünen Beinkleidern mit gelben Vertikalstreifen und spitzen braunen Schuhen läuft mit aufgerichtetem Speer einem gefleckten Panther (?) entgegen. Der nächste - in grüner Tunika, mit fleischfarbenen Hosen mit grünem Mittelstreifen und dunkelbraunen Schuhen - läuft einem Jagdhund hinterher, den er an einer rosa Leine führt (Abb. 6). Jenseits eines Baumes mit Laub und kugeligen Früchten kommt ihnen ein grauer Hase entgegen, der von einem weiteren, gelbbraunen Hund auf sie zugehetzt wird (Abb. 7).

Aus der Frieszone unterhalb des Hylasbildes haben sich nur Tiere, keine Eroten, erhalten: Links sieht man die Reste eines nach rechts laufenden Tigers, der einem nicht mehr erkennbaren Tier nachsetzt. Nach einer großen Lücke kommt ein gelbliches Raubtier (ein Hund?), welches ein gehörntes Wild, wohl eine Wildziege, verfolgt.

In der Rahmenzone oberhalb des Hylasbildes sieht man noch einen nach links laufenden Eroten in weißer Tunika, grünen Beinkleidern mit roten und rosa Streifen mit Speer und Schild (Abb. 8). Obwohl vor (= links von) ihm ein großer grauer Paarhufer mit langem Schwanz nach links rennt, scheint er den Angriff eines anderen Tieres, das von hinten kommt, abzuwehren. Zwischen ihm und dem Tier befindet sich ein Baum.

Eine Verbindung von Hylasraub und Eroten findet sich auch auf dem Mosaik der Maison à la Mosaïque de Vénus in Volubilis ${ }^{61}$ : Dort sind in zwei seitlichen Streifen Eroten in Amphitheaterszenen gezeigt. Es läßt sich allerdings keine unmittelbare inhaltliche Verbindung zwischen den beiden Darstellungen erkennen. Vielmehr war die Abbildung von Eroten oder Kindern bei grausamen, in unseren Augen für jene gänzlich ungeeignet erscheinenden Aktivitäten wie der Jagd, der venatio im Zirkus oder gar dem Gladiatorenkampf ein beliebtes Sujet der häuslichen Bilderwelt ${ }^{62}$. Während in den öffentlich-re-

60 Daß Friese mit jagenden Eroten keineswegs eine kontinuierliche Bewegungsrichtung der Figuren aufweisen mußten, führt uns das Mosaik mit der sog. Krönung der Aphrodite aus Seleukeia/Zeugma vor Augen: M. ÖNAL, Mosaics of Zeugma, Istanbul 2002, 32 Abb. S. 33.

61 Lancha a. O. Abb. 8; Muth a. O. 108. 132.

62 z. B. Seleukeia/Zeugma: vgl. Anm. 60 (Eroten bei der Jagd) - Karthago, Mosaik in der Maison des Chevaux: J. W. SAlomonson, La mosaïque aux chevaux de l'antiquarium de Carthage, Études d'Archéologie et d'Histoire Ancienne Publiées par l'Institut Historique Néderlandais de Rome I, Den Haag 1965, Abb. 2-3. 4a-c. Taf. 22-24 (Kinder und Eroten auf der Jagd). - Karthago, Mosaik im Triclinium eines römischen Hauses nördlich des Hafens: SALomonson a. O. 35 (Kinder als venatores im Zirkus). - Piazza Armerina, Villa, Raum 37: S. BRown, Death as Decoration. Scenes from the Arena on Roman Domestic Mosaics, in: A. Richlin (Hg.), Pornography and Representation in Greece and Rome, New York - Oxford 1992, 180-211 bes. 200 f. Abb. 9, 8; G. V. GENTILI, La villa Erculia di Piazza Armerina. I mosaici figurativi, Rom 1959, Taf. 42 (Kinder als Jäger). - Milet, Orpheusvilla, Mosaik im Triclinium: I. KRISELEIT, Antike Mosaiken. Altes Museum. Pergamonmuseum, Mainz 2000, 28-35 Abb. 24. 27. 36-42 (ja- 
präsentativen Räumen oft „echte“ Amphitheater- oder Jagdszenen dargestellt wurden, teilweise mit sehr drastischen Details wie spritzendem Blut und getöteten Kämpfern ${ }^{63}$, gelang in den privateren, dem otium dienenden Bereichen des Hauses durch Kinder oder Eroten eine spielerische Umsetzung von Themen des Alltagslebens: Während Kinder (Knaben) bei der Jagd auf kleine Tiere oder bei Wagenrennen auf die Erwachsenenwelt vorausweisen, eigneten sich Eroten, die außer bei der Jagd oder im Zirkus auch beim Fischfang ${ }^{64}$, bei der Ernte oder Weinlese ${ }^{65}$ gezeigt wurden, zur Entrückung des Alltäglichen in eine unrealistische, humoristische Sphäre. In beiden Fällen sollte der heiterentspannten Atmosphäre der aus der Öffentlichkeit zurückgezogenen Räume des Hauses Rechnung getragen werden ${ }^{66}$.

Eine Datierung des Mosaiks von Nauloi ist aufgrund der fehlenden archäologischen Evidenz äußert schwierig. Immerhin läßt sich die Entstehung aber anhand des ikonographischen Typus der Hylasdarstellung nach oben eingrenzen. Die Körperhaltung des Helden, der mit einem Bein auf einem Felsen kniet, das andere seitlich zu Boden gestreckt hat ${ }^{67}$, ist auf den pompejanischen Wandmalereien sowie auf den Mosaiken und Stuckreliefs des 1. Jahrhunderts n. Chr. noch nicht bekannt, wird aber später verbindlich. Alle bekannten Hylasmosaiken der mittleren und späten Kaiserzeit zeigen die Szene des Hylasraubes in dieser Weise. Deshalb kann eine Entstehung des Mosaiks aus Nauloi vor dem 2. Jahrhundert ausgeschlossen werden.

Keine Relevanz für die Datierung scheint im Gegensatz dazu die Zahl der abgebildeten Nymphen gehabt zu haben, die stets sehr variabel gehandhabt wurde $^{68}$.

Anhaltspunkte für die Zeitbestimmung des Paviments lassen sich aufgrund fehlender anderer Evidenzen schließlich nur über den relativ unsicheren Weg der Stilkritik gewinnen. Wesentliche Kriterien dafür sind traditionell die Wiedergabe der Körper hinsichtlich ihrer Form, Haltung, Bewegung, Plastizität und ihres Verhältnisses zum Hintergrund.

An den erhaltenen Oberkörpern der Nymphen sowie am erhobenen Arm des Hylas zeigt sich, daß man bemüht war, durch Farbschattierungen und vor

gende Eroten). - Zu weiteren Darstellungen von Eroten auf der Jagd vgl. LIMC III 1 (1986) 952-1049 s. v. Eros/Amor-Cupido [N. Blanc - F. Gury] bes. 991-994 Nr. 296326 .

63 z. B. Zliten, Villa von Dar Buc Amméra: Dunbabin, Mosaics of Roman North Africa, 278 Zliten 1e Taf. I Abb. 1; XX Abb. 47. 48. - Silin, Villa: V. Omar AL-MahJub, in: III Coloquio Internazionale sul Mosaico Antico, Ravenna 1980 (1983) 303f. Abb. 8 Farbtaf. - Thysdrus, Sollertiana Domus: Dunbabin a. O. 259 El Djem 21 b Taf. XXI Abb. 50 f. - Smirat bei Thysdrus, Mosaik mit Amphitheaterszenen: K. M. D. DunBabin, Mosaics of the Greek and Roman World, Cambridge 1999, Abb. 118.

64 LIMC III 1 (1986) Nr. 433-448; für Nauloi s. u. 196.

65 Ebenda Nr. 480-520.

66 Eine Anspielung auf Erotik in Verbindung mit den mythologischen Liebesszenen erscheint mir ebenso wie die von LaNCHA (ausgehend von Volubilis) vorgeschlagene Betonung der Bestrafungs- bzw. Todesthematik nicht zwingend, vgl. MuTH a. O. 133.

67 Obwohl der untere Teil des Hylasbildes fehlt, ist aufgrund der sichtbaren Partien nur eine derartige Rekonstruktion des Gesamten möglich.

68 Die Dreizahl wie in Nauloi findet sich z. B. auf den pompejanischen Wandmalereien, auf dem Wandbild der sog. Basilika von Herculaneum (LIMC a. O. Nr. 11*; LING a. O. Taf. 4), auf den Mosaiken von Tor Bella Monaca, Cuicul, Amphipolis, Carranque und Italica, auf dem opus-sectile-Bild in der Basilika des Iunius Bassus sowie auf Reliefdarstellungen des 2. und 3. Jahrhunderts (LIMC a. O. Nr. 21*. 22. 23. 24*). - In der Literatur existieren seit Apollonios Rhodios und Theokrit unterschiedliche Überlieferungen zur Anzahl der Nymphen (eine bzw. drei). 
allem helle Glanzlichter ein plastisches Erscheinungsbild zu erzeugen (Abb. 9). Durch den großflächigen Farbauftrag mit schwachen chromatischen Abstufungen sowie die harten Körperkonturen ist diesen Bestrebungen allerdings nur mäßiger Erfolg beschieden. Auch wirken die Figuren trotz der natürlichen Proportionierung von Kopf und Rumpf recht steif, die Bewegungen sind trotz der intendierten Heftigkeit des Geschehens moderat. Die sichtbaren Teile der Körper sind vor einen undifferenzierten weißen Hintergrund gestellt, wobei die Landschaftsangaben an den Bildrändern mehr als Hinweise auf den Ort des Geschehens denn als richtige Kulissen aufgefaßt sind. Die Eroten- und Tierfiguren der Bordüre zeigen eine geradezu zeichnerische Auffassung mit dunklen Binnen- und Konturlinien. Im Gegensatz dazu ist die Akanthusranke durch weiße Glanzlichter geradezu übertrieben plastisch gestaltet $^{69}$.

Obwohl man sich der Relativität der Aussagekraft von stilistischen Beobachtungen bewußt sein muß, da diese nicht unbedingt zeitliche Entwicklungen, sondern vor allem das Können der ausführenden Werkleute veranschaulichen, lassen die genannten Merkmale eher auf eine Entstehung im 3. als im 2. Jahrhundert n. Chr. schließen. Zwar sind die Bestrebungen, durch Farbgebung und Licht-Schatten-Effekte Plastizität der Körper zu erzeugen, auf dem Mosaik von Nauloi nicht ausgereift, doch muß man für sie die Entwicklungen des flavischen Kolorismus sowie des antoninisch-severischen Hell-DunkelIllusionismus voraussetzen ${ }^{70}$. Wie weit nach unten man die Entstehung des Mosaiks annehmen darf, läßt sich beim jetzigen Forschungsstand nicht einwandfrei festlegen, zumal die Mosaikkunst Ostpamphyliens bzw. des unmittelbar benachbarten Westkilikiens noch nicht ausreichend erforscht ist und daher lokale Vergleichsbeispiele fehlen ${ }^{71}$. Einmal mehr zeigt sich, daß nicht aufgrund weitgespannter Stilvergleiche verbindliche Aussagen zur Datierung eines Kunstwerks möglich sind, sondern daß vielmehr die Untersuchung von örtlichen Werkstätten forciert werden muß.

69 Eine Reihe von Mosaiken aus dem Südosten der heutigen Türkei besitzt dunkelgrundige Bordüren mit Akanthusranken. Vielfach sind sie wie hier mit allerlei Früchten und Blüten gefüllt, oft sogar von Tieren, Maskenköpfen und/oder Eroten bevölkert; vgl. z. B. ein Mosaik aus Tarsos, wahrscheinlich aus der Mitte des 3. Jahrhunderts: L. BudDE, Antike Mosaiken in Kilikien II, Recklinghausen 1972, 125 Abb. 118-121. 124-126. 129-131; RBK IV (1990) 319-344 s. v. Kommagene - Kilikien - Isaurien IV. Mosaiken [G. Hellenkemper SAlies] bes. 320; Adana, Mosaik mit Bestrafung der Dirke: Budde a. O. 25-27 Abb. 31: Anfang 4. Jahrhundert; Hellenkemper Salies a. O.: zweite Hälfte 3. Jahrhundert; Seleukeia/Zeugma, Mosaik der „Synaristosai“: ÖNAL, Zeugma (wie Anm. 60) 60 f.: 2. - Mitte 3. Jahrhundert; Mosaik mit Eros und Psyche: ÖNAL, a. O. 27-29: 2. - Mitte 3. Jahrhundert; Antiocheia, Mosaik aus Raum 1 der Constantinian Villa: D. Levi, Antioch Mosaic Pavements, Princeton 1947, 129. - Nur bei den beiden letztgenannten Mosaiken sind die Ranken ähnlich plastisch gestaltet wie in Nauloi.

70 Die grundlegende Untersuchung zur Entwicklung des Figurenstils auf Mosaiken stammt noch immer von D. Levi, Antioch Mosaic Pavements, $518 \mathrm{ff}$. - In Grundzügen übernommen wurde sie von J. BALty, La mosaïque antique au Proche-Orient I. Des origines à la Tétrarchie, in: ANRW XII 2 (1984) 347-429.

71 Für Pamphylien liegen bisher keine Überblickswerke zur Mosaikkunst vor; Einzelpublikation finden sich vor allem in türkischen Periodika. - Zu den Mosaiken Kilikiens vgl.: BudDE, Antike Mosaiken in Kilikien I-II, Recklinghausen 1969-1972; BALTY a. O. 404-409; J. Russell, The Mosaic Inscriptions of Anemurium, ETAM 13, Dph ÖAW 190, Wien 1987; Hellenkemper SAlies a. O.; S. CAMPBell, The Mosaics of Anemurium, Subsidia Mediaevalia 25, Toronto 1998. 
Bei der Frage nach möglichen Werkstattzusammenhängen mit anderen Mosaiken aus der Gegend von Syedra komme ich im Anschluß auf die bisher aus der Stadt selbst bekannt gewordenen Exemplare zu sprechen.

In der Ober- und Unterstadt wurden im Zuge der durchgeführten Surveys einige Mosaiken festgestellt. Bei allen Exemplaren handelte es sich um Reste, meist von Randbordüren, die vielfach aufgrund rezenter Zerstörungen heute im Gelände nicht mehr sichtbar sind ${ }^{72}$ : bäuden:

Aus der Oberstadt (Syedra) kennt man Mosaik(böd)en aus folgenden Ge-

- Aus dem Südschiff der frühchristlichen Kirche I. 8. C stammt ein Fußbodenmosaik, „das auf weißer Grundfläche eine Grenzlinie und ein Flechtoder Rankenmuster in dunkelblauem Linienzug sowie eine rote Blatt- oder Herzform aus Ziegel-Tesserae (Größe 12-15mm)“ hatte ${ }^{73}$.

- Bei II. 3, westlich unterhalb der Stadtmauer, in einem ungeklärten Gebäudezusammenhang, fanden sich Reste eines Mosaiks mit dunkelblauer Randzone, an die eine weiße Zone mit einem geometrischen Muster anschloß. Wie bei der frühchristlichen Kirche war dem Setzmörtel Ziegelmehl beigemengt ${ }^{74}$.

- Im Thermengymnasium III. 17, dem dominierenden Bau der Ruinen von Syedra, fand sich in Raum E (frigidarium) ein einfacher weißer Mosaikboden aus groben Tesserae (b-10-13 mm), die in Ziegelsplittmörtel gesetzt waren $^{75}$.

Aus der Unterstadt (Nauloi) kamen außer dem hier behandelten Mosaik im Bereich VII. 6 neben verstreuten Architekturteilen aus Haustein und Dachziegeln Bruchstücke eines Tessellats zum Vorschein, dessen Würfel $\left(>1 \mathrm{~cm}\right.$ ) einen Randstreifen und ein geometrisches Motiv bildeten ${ }^{76}$.

Zuletzt wurde 1994 im Zuge einer Notgrabung im Gebiet der Unterstadt, in der Flur Adataş, ein noch gut erhaltenes Mosaik gefunden, das aus einer Badeanlage stammen dürfte: Es war in einem Raum verlegt, dessen Nord-, Süd- und Westmauer noch 50-60 cm hoch erhalten waren, während an der Ostseite nur mehr ein Loch existierte, das Einblick in ein darunterliegendes Gewölbe gewährte ${ }^{77}$. Der 3,50×2,80 m messende Mosaikboden, dessen Ostteil zerstört war, ist aus $1 \times 1 \mathrm{~cm}$ großen Tesserae in den Farben Weiß, Blau, Gelb und Grün zusammengesetzt. Er zeigt im Mittelbild eine marine Szene: Zwei nackte Eroten befinden sich in einem Nachen, der eine rudert, der andere hat die Angel ausgeworfen. Rund um das Boot sind im Wasser ein Thunfisch, ein Aal, ein Schwertfisch, ein Tintenfisch sowie ein Oktopus zu sehen ${ }^{78}$. Das Meeresbild wird durch eine Bordüre mit dunklem Astragal auf weißem Grund gerahmt. Heute befindet sich das restaurierte Mosaik in einer eigens dafür geschaffenen Pergola im Garten des Archäologischen Museums von Alanya. Vom Ausgräber wurden bisher keine Vorschläge zur Datierung gemacht, hierfür ist die Publikation abzuwarten. Aufgrund der publizierten Bil-

\footnotetext{
72 Huber, Syedra passim.

73 Ebenda 47.

74 Ebenda 51.

75 Ebenda 67.

76 Ebenda 78.

77 İ. KaRAmUt, 1994 Yılı Syedra Antik Kenti Çevre Düzenleme Çalışmaları, MKKS 6, 1995 (1996) 82 Abb. 8. 9.

78 Ebenda.
} 
der läßt sich aber jetzt schon feststellen, daß keine Bezüge zum Hylasmosaik vorhanden sind.

\section{ABBILDUNGSVERZEICHNIS \\ (Alle Bilder G. Huber)}

Abb. 1: Mittelbild mit der Szene des Hylasraubes, umgeben von der Akanthusbordüre

Abb. 2: Akanthusbordüre. Detail

Abb. 3: Jagdfries rechts

Abb. 4: Hylas wird von den Nymphen geraubt

Abb. 5: Jagdfries rechts und oben

Abb. 6: Jagdfries rechts. Detail

Abb. 7: Jagfries rechts. Detail

Abb. 8: Jagdfries oben. Detail

Abb. 9: Detailansicht der linken Nymphe.

Abb. 10: Detailansicht der rechten Nymphe. 


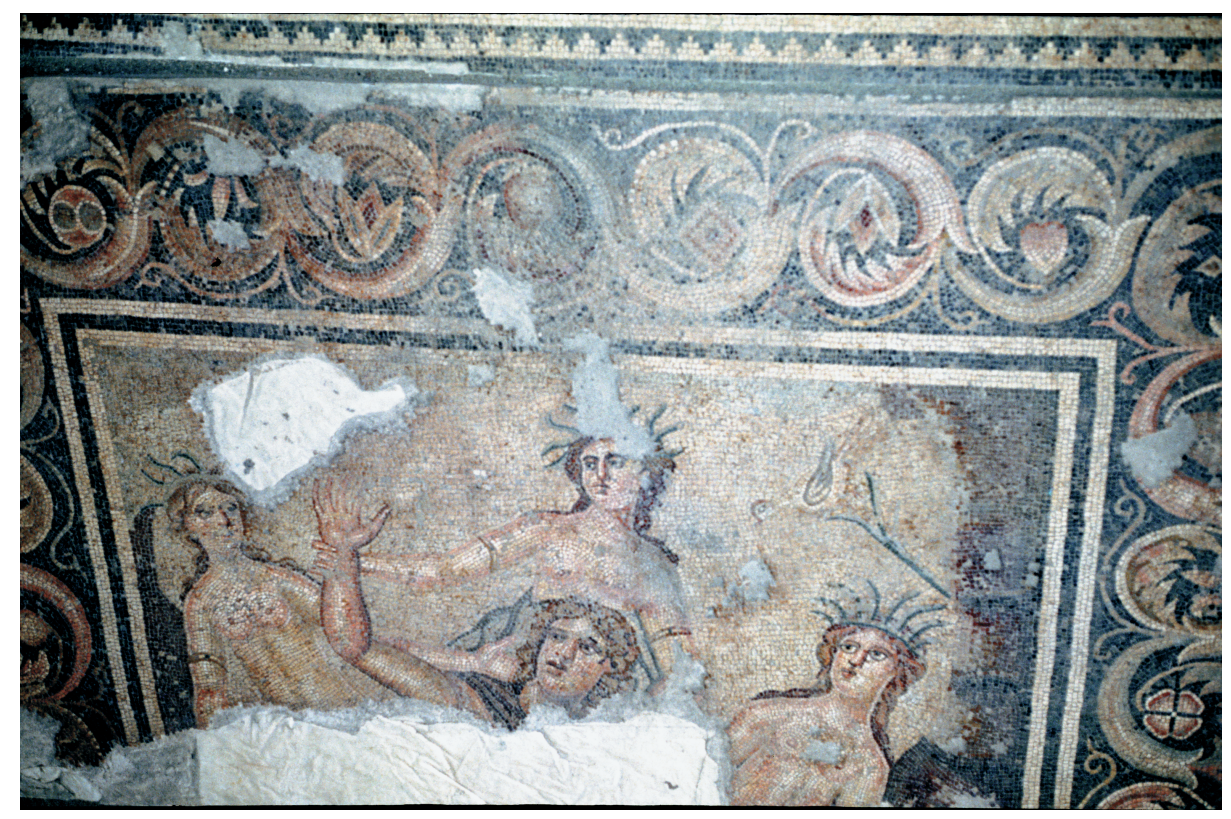

Abb. 1: Mittelbild mit der Szene des Hylasraubes, umgeben von der Akanthusbordüre

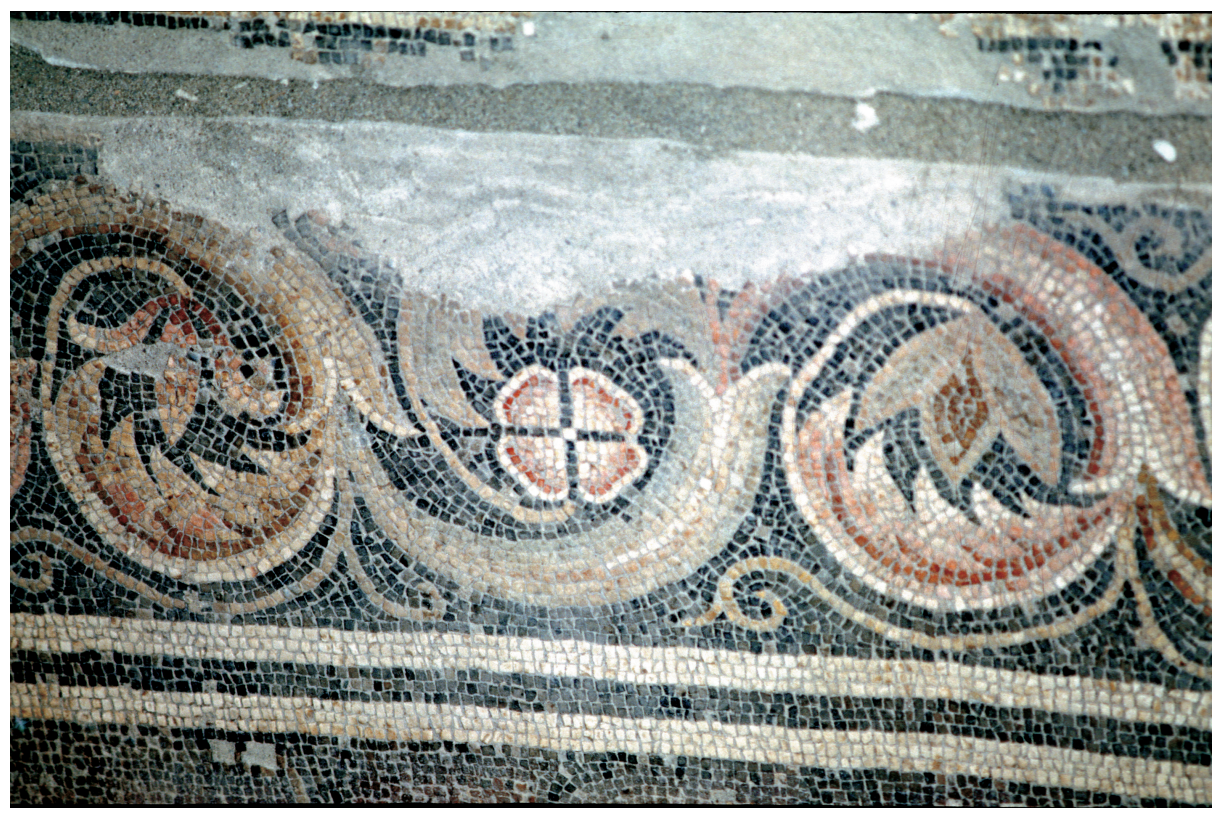

Abb. 2: Akanthusbordüre. Detail 


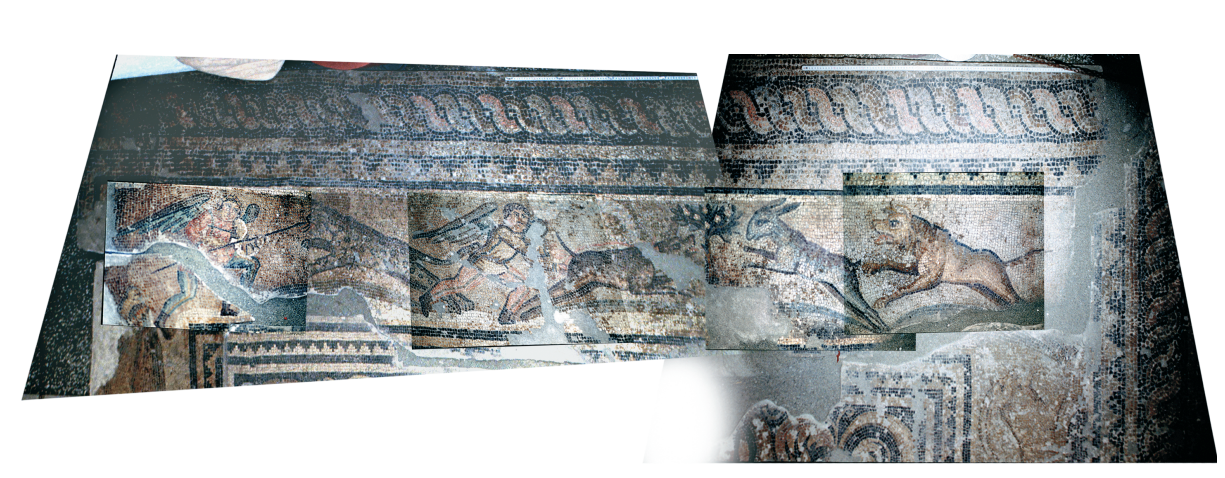

Abb. 3: Jagdfries rechts

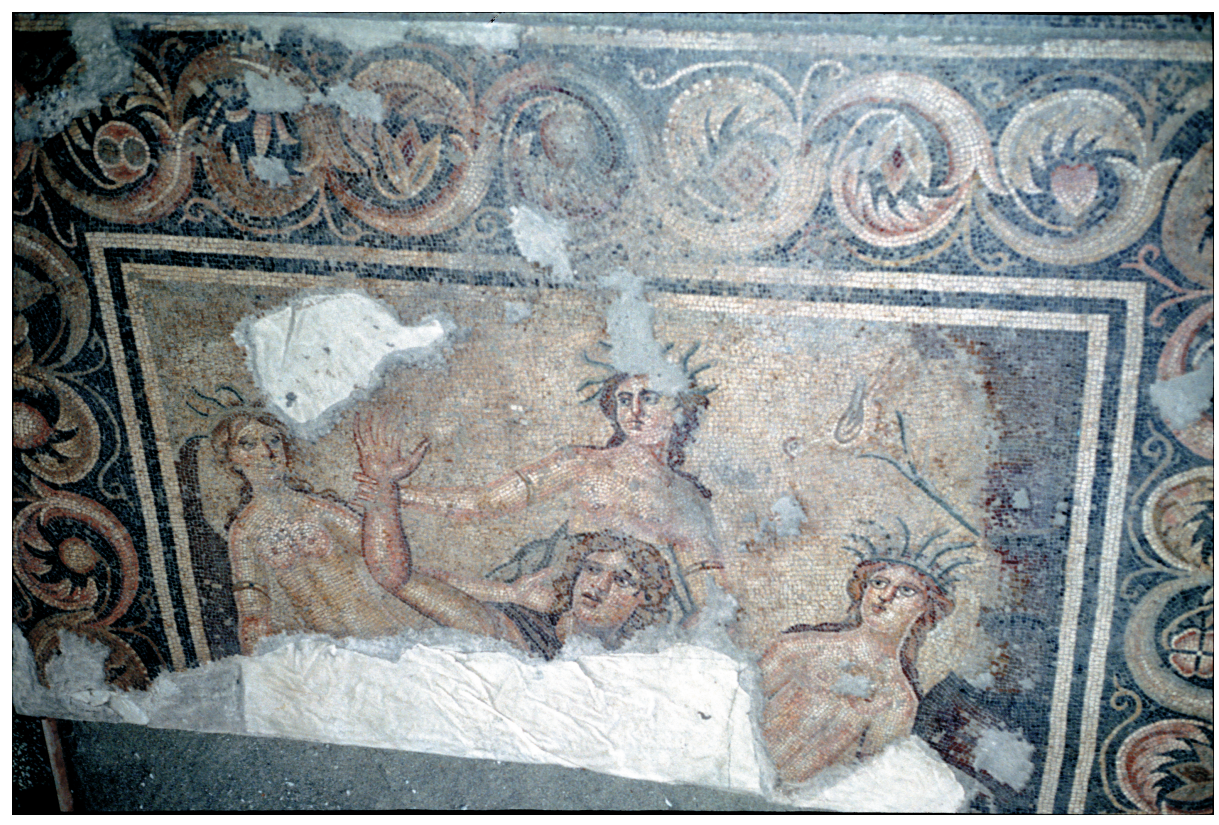

Abb. 4: Hylas wird von den Nymphen geraubt 


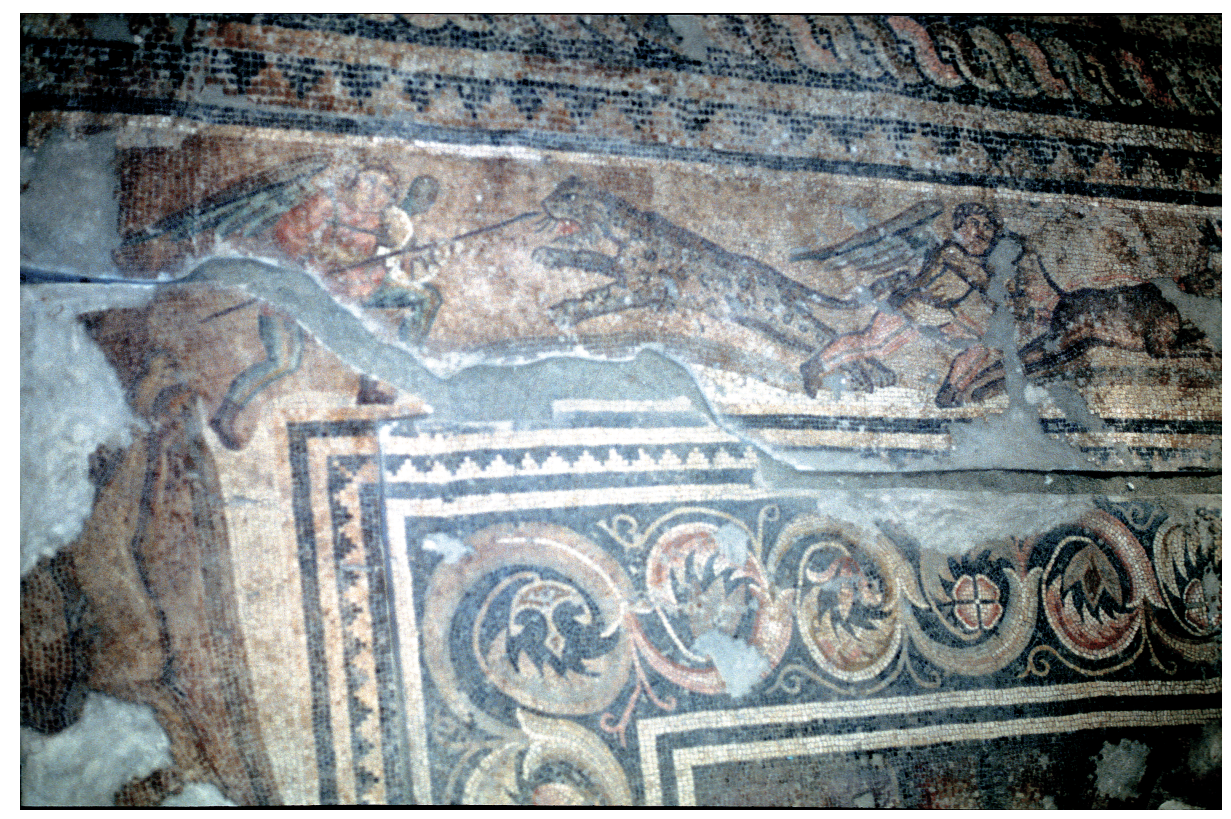

Abb. 5: Jagdfries rechts und oben

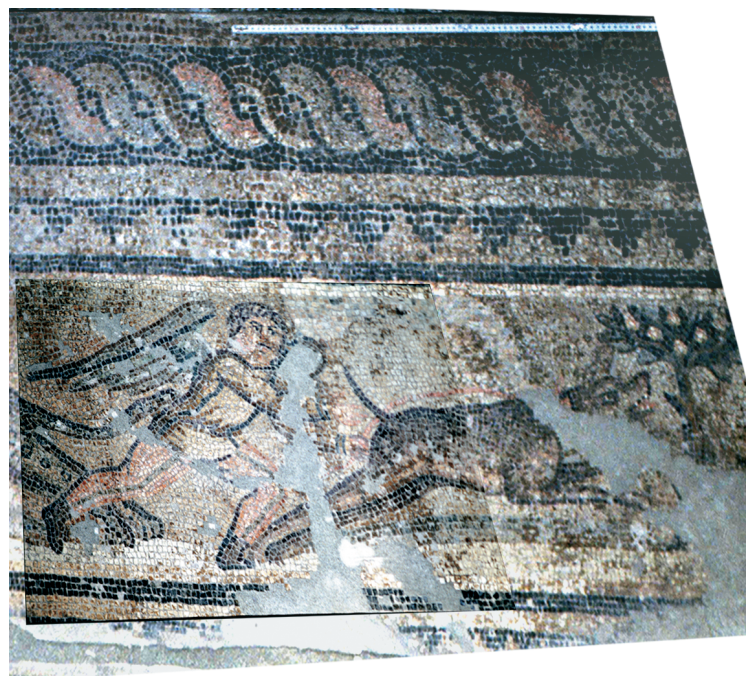

Abb. 6: Jagdfries rechts. Detail 


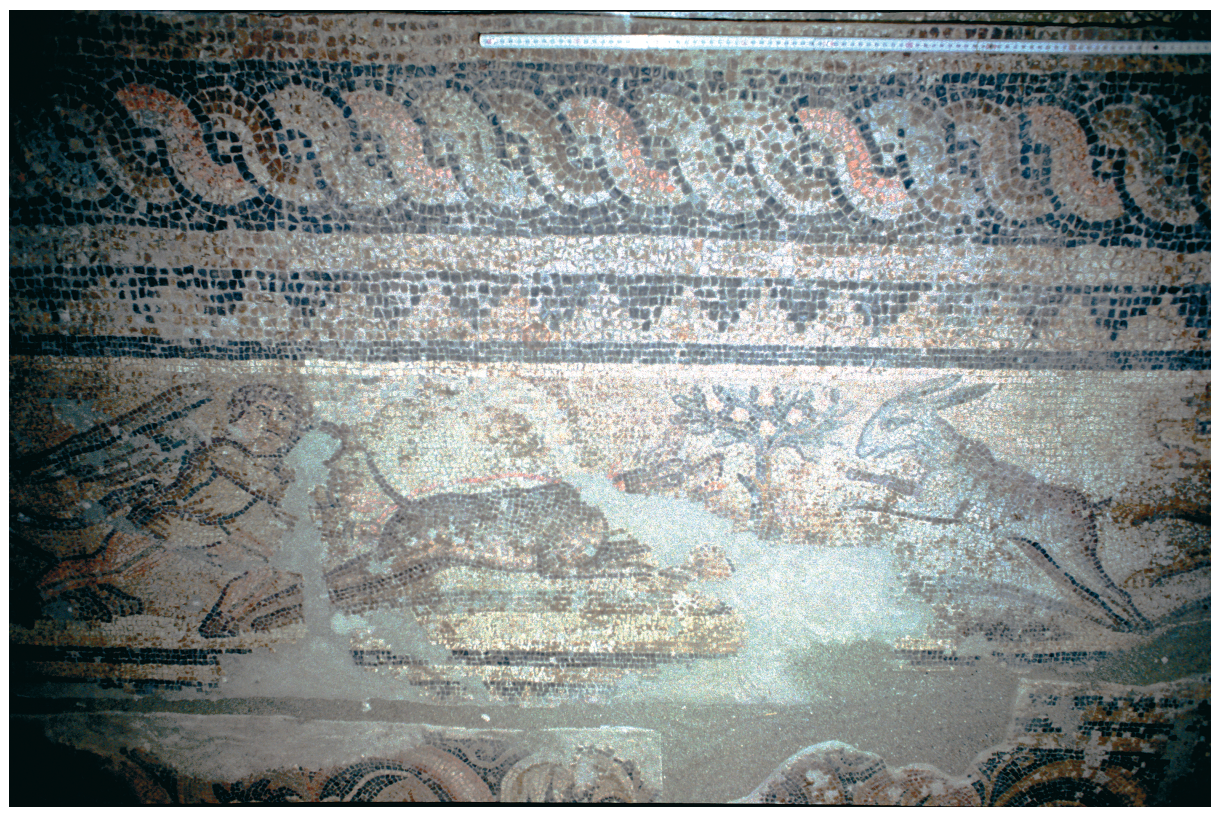

Abb. 7: Jagfries rechts. Detail

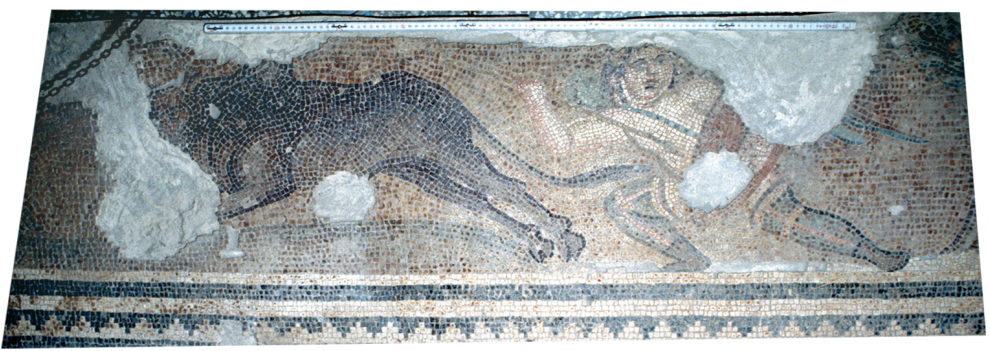

Abb. 8: Jagdfries oben. Detail

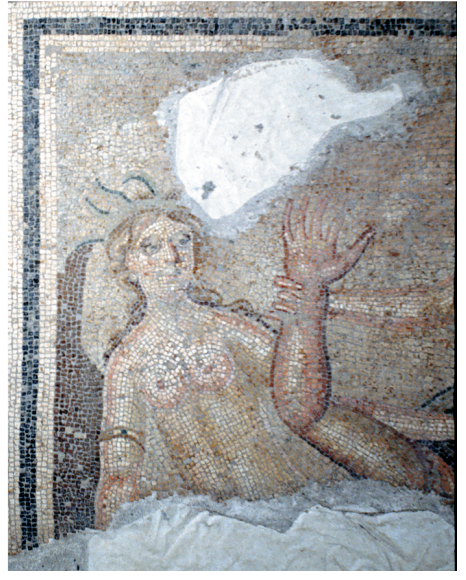

Abb. 9: Detailansicht der linken Nymphe. 


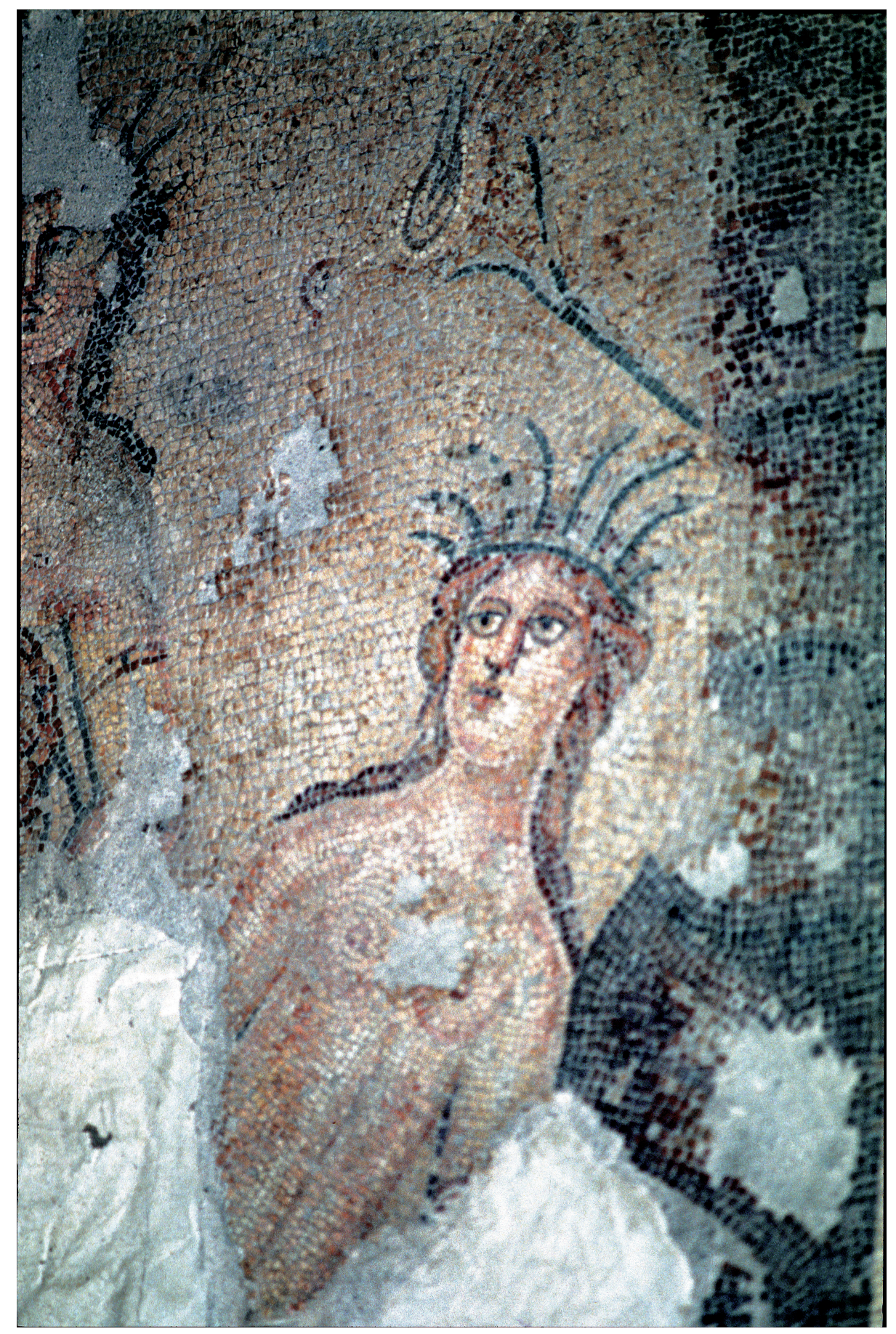

Abb. 10: Detailansicht der rechten Nymphe. 